\title{
Therapie in der Schwangerschaft
}

\author{
Therapy in Pregnancy
}

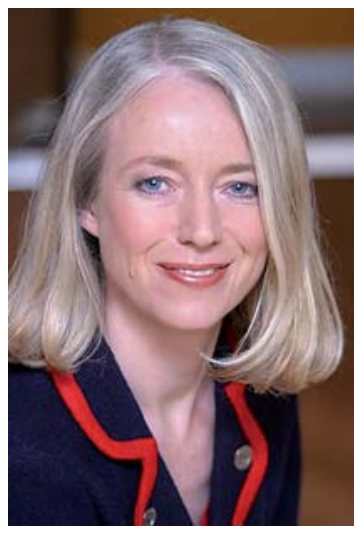

Prof. Dr. Christiane Bayerl
Der CME-Artikel in diesem Heft beschreibt eindrucksvoll Dermatosen in der Schwangerschaft. Auch die Therapie in der Schwangerschaft ist eine Herausforderung. Physiologische Vorgänge zwischen dem Erwachsenen- und dem Säuglingsorganismus unterscheiden sich. Zudem sind die transdermale Penetration und die nachfolgende systemische Bioverfügbarkeit der verschiedenen topischen Aknezubereitungen wenig untersucht [1]. Die ärztliche Aufgabe ist dann, zwei Organismen, Mutter und ungeborenem Kind/Säugling, in Schwangerschaft und Stillzeit zu nützen oder zu helfen, ohne einem der beiden zu schaden.

Studien zum Medikamenteneinsatz in Schwangerschaft und Stillzeit wird es auch in Zukunft nicht geben. Umso wichtiger ist es, die Patientin im Gespräch darauf hinzuweisen, dass sie den Plan schwanger zu werden mitteilt. Bei einer eingetretenen Schwangerschaft unter aktuell laufender Therapie muss kommuniziert werden, dass sie sich erneut beim Dermatologen/behandelnden Arzt vorstellt. Ebenso wichtig ist, dass ärztlicherseits bei jeder Konsultation und entsprechender Therapie eine Schwangerschaft nachgefragt wird. Die Vergleichbarkeit mit vorausgegangenen Medikamentenstudien ist nur eingeschränkt möglich, da die physiologischen Veränderungen in der Schwangerschaft pharmakologische Folgen haben, z.B. ist ein Medikament in der Schwangerschaft länger im Gastrointestinaltrakt. Die Plasmaproteinkonzentration reduziert sich und damit steigt die Menge des ungebundenen bioaktiven Medikaments im Organismus. Andererseits nimmt das Volumen des Blutes in der Schwangerschaft zu und die potenziellen Serumspiegel eines Medikamentes sinken. Aufgrund des osmotischen Gradienten werden Substanzen, die die Plazenta schlecht passieren, in signifikanten Konzentrationen im Fetus angereichert. 3-5\% kongenitaler Anomalien sind Folge einer teratogenen Substanz (z.B. Thalidomid), die die Mutter als Medikament zu sich nahm.

Nehmen wir als Beispiel die Akne, ein Krankheitsbild der ausgehenden Pubertät, das auch in eine Zeit der Schwangerschaft fallen kann. Am wichtigsten ist, wenn zum Zeitpunkt der Konzeption eine topische Therapie mit Retinoiden durchgeführt wurde, diese sofort zu beenden. Ein Schwangerschaftsabbruch ist jedoch nicht erforderlich. Kontrollierte Studien zu Aknetherapeutika in Schwangerschaft und Stillzeit existieren nicht [2,3].

Tetrazykline, Doxyzyklin, Minozyklin oder Isotretinoin sind nicht einzusetzen. Topisches Benzoylperoxid und topische Azelainsäure sind sichere Therapiemöglichkeiten. Eine systemische Therapie kann bei ausgeprägt schwerer Akne nach den
Leitlinien [4] auch in der Schwangerschaft mit Glukokortikoiden ab dem zweiten Trimenon in Zusammenarbeit mit dem Gynäkologen und/oder Erythromycin (letzteres nicht in der Stillzeit) durchgeführt werden. Zink kann, falls nötig, oral gegeben werden, jedoch nicht länger als 3 Monate [5]. Ist denn dann zumindest das Angebot aus der kosmetischen Dermatologie möglich? Ein chemisches Peeling mit alpha-Hydroxy-Säuren ist eine ergänzende Option zur Behandlung in Schwangerschaft und Stillzeit, auch wenn Studien fehlen [4]. Salizylsäure-Peelings [6,7], aber auch Trichloressigsäure-Peelings oder Phenol-Peels verbieten sich aufgrund der systemischen Aufnahme. Ergänzende kosmetologische Maßnahmen zur medizinisch dermatologischen Aknetherapie sind gerade in der Schwangerschaft sinnvoll, wenn die Indikation dermatologisch gestellt und die Maßnahme dermatologisch begleitet wird. Zu diesen ergänzenden Maßnahmen zählen die Reinigung und Pflege der zu Akne neigenden Haut, das manuelle Ausreinigen und Camouflage auch in Ergänzung zur medikamentös behandelten Aknehaut [8].

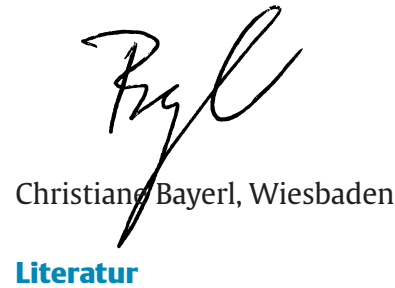

1 Krautheim A, Gollnick H. Transdermal penetration of topical drugs used in the treatment of acne. Clin Pharmacokinet 2003; 42: 1287-1304

2 Akhavan A, Bershad S. Topical acne drugs: review of clinical properties, systemic exposure, and safety. Am J Clin Dermatol 2004; 4: 473-492

3 Rothman KF, Pochi PE. Use of oral and topical agents for acne in pregnancy. J Am Acad Dermatol 1988; 19: $431-442$

4 Nast A, Bayerl C, Borelli C et al. S2k-guideline for therapy of acne. JDDG 2010; 8 (Suppl. 02): S1 - 59

5 Bayerl C. Acne therapy in pregnancy. Hautarzt 2013; 64: 269-273

6 Cosmetic Ingredient Review Panel. Safety assessment of Salicylic acid, Butyloctyl Salicylate, Calcium Salicylate, C12-15 Alkyl Salicylate, Caproyl Salicylic Acid, Hexyldodcyl Salicylate, Isocetyl Salicylate, Isodecyl Salicylate, Magnesium Salicylate, MEA-Salicylate, Athylhexyl Salicylate, Potassium Salicylate, Methyl Salicylate, Myristyl Salicylate, Potassium Salicylate, Methyl Salicylate, Myristyl Salicylate, Sodium Salicylate, and Tridecyl Salicylate. Int J Toxicol 2003; 22: 1 108

7 Sasseville $D$. Dermatological therapy during pregnancy and lactation. In: Harahap M, Wallach RC, eds. Skin Changes and Disease in Pregnancy. New York: Marcel Dekker; 1996: 249-319

8 Bayerl C, Degitz K, Meigel E, Kerscher M. Adjuvant dermato-cosmetic acne therapy. JDDG 2010; 1: S89-94 